\title{
The OMERACT Core Set of Domains for Outcome Measures in Behçet Syndrome
}

Gülen Hatemi, Alexa Meara, Yesim Ozguler, Haner Direskeneli, Alfred Mahr, Beverly Shea, Esen Cam, Ahmet Gul, Yusuf Yazici, Peter Tugwell, Hasan Yazici, Peter A. Merkel, for the OMERACT Behçet Syndrome Working Group.

G. Hatemi, MD. Professor of Medicine, Division of Rheumatology, Department of Internal Medicine and Behçet's Disease Research Center, Istanbul University - Cerrahpasa, Istanbul, Turkey

A. Meara, MD. Assistant Professor of Medicine, Division of Rheumatology and Immunology. Ohio State University, Columbus, Ohio, USA

Y. Ozguler, MD. Division of Rheumatology, Department of Internal Medicine and Behçet's Disease Research Center, Istanbul University - Cerrahpasa, Istanbul, Turkey

H. Direskeneli, MD. Professor of Rheumatology, Division of Rheumatology, Department of Internal Medicine, Marmara University, Faculty of Medicine, Istanbul, Turkey

A. Mahr, MD, PhD, Professor of Internal Medicine, Clinic for Rheumatology, Cantonal Hospital St. Gallen, St. Gallen, Switzerland

B. Shea, PhD, MSc, BScN, RN, Adjunct Professor, Division of Rheumatology, Department of Medicine, and School of Epidemiology and Public Health, Faculty of Medicine, University of Ottawa, and Clinical Epidemiology Program, Ottawa Hospital Research Institute, Ottawa, Canada

E. Cam, Patient-Partner, Istanbul, Turkey

A. Gul, MD. Professor of Rheumatology, Division of Rheumatology, Department of Internal Medicine, Istanbul University, Istanbul Faculty of Medicine, Istanbul, Turkey

Y. Yazici, MD. Associate Professor of Medicine, New York University School of Medicine, New York, New York, USA

P. Tugwell, MD. Professor of Medicine, Department of Medicine, University of Ottawa, Ottawa, Canada

H. Yazici, MD. Professor of Rheumatology, Academic Hospital, Istanbul, Turkey

This article has been accepted for publication and undergone full peer review but has not been through the copyediting, typesetting, pagination and proofreading process, which may lead to differences between this version and the Version of Record. Please cite this article as doi: 10.1002/acr.24511

This article is protected by copyright. All rights reserved 
P.A. Merkel, MD, MPH, Professor of Medicine and Epidemiology, Division of Rheumatology and Department of Biostatistics, Epidemiology, and Informatics, University of Pennsylvania, Philadelphia, Pennsylvania, USA

\section{Corresponding Author:}

Gülen Hatemi, MD

Professor of Medicine, Division of Rheumatology, Department of Internal Medicine and Behçet's Disease Research Center, Istanbul University - Cerrahpasa

Istanbul, Turkey

gulenhatemi@yahoo.com

Keywords: Behçet, outcomes, clinical trials, OMERACT

Word Count (main text): 3799

Funding: The Vasculitis Clinical Research Consortium (VCRC) has received support from the National Institute of Arthritis and Musculoskeletal and Skin Diseases (U54AR057319), the National Center for Research Resources (U54RR019497), the Office of Rare Diseases Research, and the National Center for Advancing Translational Science (NCATS). The VCRC is part of the Rare Diseases Clinical Research Network, a program of NCATS.

Disclosures: Dr. Hatemi reports receiving funds for the following activities: Lecture fees and fee for serving on an advisory board from Celgene, consulting fees from UCB Pharma, Bayer, Johnson \& Johnson, lecture fees from Novartis, Abbvie, UCB Pharma. Dr Direskeneli reports receiving funds for the following activities : PI for Apremilast Study for Cellgene, educational funds from AbbVie, Pfizer, MSD, Roche, UCB, Amgen, Novartis. Dr. Mahr has served as a speaker for Chugai and Roche and as a consultant for Celgene. $\operatorname{Dr}$ Y Yazici reports the following activities: Consulting: Amgen, BMS, Sanofi, Celgene. Research support: Celgene, BMS, Genentech. Dr. Tugwell receives funds from the following UCB [Data Monitoring Committee), Canadian Reformulary Committee, and UpToDate. Dr. Merkel reports receiving funds for the following activities: Consulting: AbbVie, AstraZeneca, Biogen, Boeringher-Ingelheim, Bristol-Myers Squibb, Celgene, ChemoCentryx, CSL Behring, Genentech/Roche, Genzyme/Sanofi, GlaxoSmithKline, InflaRx, Insmed, Jannsen, Kiniksa, Sparrow; Research Support: AstraZeneca, Boeringher-Ingelheim, Bristol-Myers

This article is protected by copyright. All rights reserved 
Squibb, Celgene, ChemoCentryx, Genentech/Roche, Genzyme/Sanofi, GlaxoSmithKline, InflaRx, Kypha, TerumoBCT; Royalties: UpToDate.

This article is protected by copyright. All rights reserved 


\section{ABSTRACT}

Objective: There is an unmet need for reliable, validated, and widely-accepted outcome measures for randomized clinical trials in Behçet syndrome (BS). The Outcome Measures in Rheumatology Clinical Trials (OMERACT) BS Working Group, a large, multi-disciplinary group of experts in BS and patients with BS, worked to develop a Core Set of data-driven outcome measures for use in all clinical trials of BS.

Methods: The Core Domain Set was developed through a comprehensive, iterative, multi-stage project which included a systematic review, a focus group meeting and qualitative patient interviews, a survey among experts in BS, a Delphi exercise involving both patients and physician-experts in BS, and utilization of the data, insight, and feedback generated by these processes to develop a final Core Domain Set.

Results: All steps were completed and domains were delineated across the organ systems involved in this disease. Since trials in BS often focus on specific manifestations and not the disease in its entirety, the final proposed Core Set includes 5 domains mandatory for study in all trials in BS (disease activity, new organ involvement, quality of life, adverse events, and death) with additional sub-domains mandatory for study of specific organ-systems. The final Core Set was endorsed at the 2018 OMERACT meeting.

Conclusion: The Core Set of Domains in BS provides the foundation through which the international research community, including clinical investigators, patients, biopharmaceutical industry, and government regulatory bodies can harmonize the study of this complex disease, compare findings across studies, and advance development of effective therapies.

This article is protected by copyright. All rights reserved 


\section{SIGNIFICANCE AND INNOVATION}

- The heterogeneity in the outcomes and outcome measures used in clinical trials of Behçet syndrome has made it difficult to compare results of trials with different agents or from different centers, conduct meta-analyses, or create combined datasets for additional analyses.

- The Core Set of Domains for clinical trials in Behçet syndrome have been developed by the Outcome Measures in Rheumatology Clinical Trials (OMERACT) Behçet Syndrome Working Group with the aim of improving disease assessment in trials of Behçet syndrome by providing a critical framework for use of outcome measures.

- The Core Set was developed through a multiyear, data-driven, iterative process following the rigorous standards of the OMERACT Filter 2.0, resulting in consensus among patients, physicians, and researchers from several countries about "what to measure" in clinical trials for Behçet syndrome. The Core Set received strong endorsement by the OMERACT community.

- An important innovation with this Core Set is that instead of a single domain set for use in all trials, there is a mandatory set of domains to be used in all trials of Behçet syndrome and separate sets of subdomains specific for each type of organ or system involvement for use in trials seeking to specifically assess that type of involvement. This approach may provide a model for outcomes assessment in other multisystem diseases.

This article is protected by copyright. All rights reserved 


\section{INTRODUCTION}

Behçet syndrome (BS) is a multi-system, variable-vessel vasculitis with a relapsing and remitting disease course with high morbidity depending on the organ system involved. It causes oral and genital ulcers, erythema nodosum-like lesions and papulopustular lesions, arthralgia or arthritis, posterior or panuveitis with retinal vasculitis, arterial aneurysms, thrombosis in arteries and veins of all sizes, parenchymal brain lesions, cerebral sinus thromboses, and intestinal ulcers (1). Skin, mucosa, and musculoskeletal involvement can be associated with significant impairment in quality of life when lesions are present without causing permanent damage, whereas ocular, vascular, nervous, and gastrointestinal system involvement can result in serious disability and may even be life-threatening. Clinical findings show important heterogeneity across patients and this makes trial design and disease assessment complicated (2).

Each patient with BS has only some of the disease manifestations over their lifetime and typically only a few of the manifestations are active at the same time (1). This lack of uniformity creates challenges in developing a therapeutic modality for the entire disease. Due to these difficulties, clinical trials for BS are usually designed to evaluate a single type of system involvement. Conducting studies in populations of patients with BS with heterogeneous manifestations using overall disease assessment instruments as primary endpoints may not be the most optimal. Some manifestations may improve while others worsen, so the change in overall disease activity scores may not be a reliable indicator of therapy efficacy. This is especially important for trials with agents that have shown differences in drug response across types of organ involvement. Variation in the frequency and severity of relapses of different organ manifestations is another challenge in overall composite disease assessment in BS. Additionally, the severity of relapses may vary, causing damage and disability in some patients and only a transient impairment of quality of life in others. Finally, impairment of function due to damage can be difficult to discern from active disease.

Although a number of trials have been conducted in BS with different agents, disease assessment has not been optimal. There is a lack of standardized outcome measures that are widely accepted and standardly used in BS trials. It has been problematic in the study of BS to compare results of randomized controlled trials with different agents or results of studies from different centers, combine results of studies with the 
same agent in meta-analyses, or combine datasets for additional analyses $(3,4)$. In summary, there are multiple challenges due to the heterogeneity of the disease that impede successful drug development.

These challenges led the Outcome Measures in Rheumatology Clinical Trials (OMERACT) Behçet Syndrome Working Group to work with a large multi-disciplinary group of experts in BS most of whom are members of the International Society for Behçet's Disease and patients with BS, to develop a Core Set of datadriven outcome measures for use in all clinical trials. The first phase of this project that has been completed and is described in this article is the Core Set of Domains for clinical trials in BS which was endorsed by OMERACT.

This article is protected by copyright. All rights reserved 


\section{MATERIALS AND METHODS}

The Core Set of Domains were created following the methodology endorsed by $\operatorname{OMERACT}(5,6)$, with the final aim of developing a Core Set of outcome measures to be used in all clinical trials in BS. This was an iterative, multi-stage, multi-year project that involved a systematic review, a survey among experts, an outcome measures focus group meeting including all stakeholders, qualitative patient interviews, a Delphi exercise involving both physician experts in BS from different specialties and countries and patients with BS, and ultimately endorsement through voting at the OMERACT 2018 meeting.

The study was approved by the Ethics Review Committee of Istanbul University Cerrahpasa Medical Faculty: 83045809/604.01

\section{Systematic review}

A systematic literature review was conducted to identify which domains were adopted as outcomes and outcome measures in previous studies of BS (7). All randomized controlled trials, non-randomized clinical trials, longitudinal or retrospective cohort studies, case series, biomarker studies, and genetic association studies that involved patients with BS were included. The domains and sub-domains that were found in the included studies were identified as candidate items for the Delphi.

\section{Interest group meeting}

To start collaborative work with a large group of experts in BS, an outcome measures special interest group meeting was held during the $16^{\text {th }}$ International Conference on Behçet's Disease (8). Multiple stakeholders were invited to this meeting. Participants included physicians and/or researchers from all specialties who were experts in the care of BS (rheumatologists, ophthalmologists, dermatologists, neurologists, gastroenterologists, oral health medicine specialists), patients with BS, and physicians from the biopharmaceutical industry experienced in designing trials for BS. The ideas and feedback generated during the meeting helped the group leaders better understand the scope of the project and shape the next steps in the project.

\section{Survey among experts}

An initial survey was conducted among experts in BS who were rheumatologists, dermatologists, ophthalmologists, gastroenterologists, internists, or dentists from 13 countries to get their opinion on the domains that need to be addressed and the instruments that are used to evaluate each of these domains in trials of BS (2). An online tool (SurveyMonkey ${ }^{\circledR}$ ) was used to conduct the survey and collect responses. A total of 51 physicians were invited by electronic mail and received up to 3 reminders. The survey 
included 11 questions about the endpoints that are relevant for trials in BS, the validity and reliability of the 4 overall disease activity assessment instruments for BS that were identified through the systematic review, the weight of potential items to be assessed in trials of BS, and whether organ-specific tools in addition to an overall disease assessment instrument are necessary to evaluate disease activity in BS.

\section{Qualitative patient interviews}

In depth, semi-structured individual patient interviews were conducted with 20 patients with BS from Turkey (15 men, 5 women, mean age $35 \pm 6$ SD years) (9). These patients were selected to represent the heterogeneous disease spectrum of BS and patients had various types of organ and system involvement. In addition to skin and mucosa lesions (all), 10 had eye involvement, 8 had vascular involvement, 6 had arthritis, 5 had nervous system involvement, 3 had gastrointestinal involvement, and 2 had only skin and mucosa involvement. Patients were interviewed about the impact of BS on their daily activities, physical function, social and family life, psychological well-being, and coping strategies. Seven conceptual components (disease onset, diagnostic experience, treatment history, disease remission, disease flare, quality of life, mental health impact) were covered using 41 open-ended questions. Interviews were audio-recorded, transcribed, translated into English, and entered into a qualitative data analysis computer software package (NVivo 11). A Grounded Theory approach was employed in thematic analysis of translated interviews $(10,11)$. In addition to better understanding the patients' perspective of $B S$, these interviews helped generate candidate domains and sub-domains important to patients that would be included in the Delphi exercise.

\section{Delphi}

The candidate domains that were retrieved through the systematic review, survey among BS experts, qualitative patient interviews and the focus group meeting among multiple stakeholder groups were incorporated into a Delphi questionnaire. Item selection for the questionnaire was influenced by the framework of OMERACT Filter 2.0 and by input from the OMERACT community (12).

Since organ systems are often studied separately in BS due to possible differences in treatment response, the questionnaire was designed in seven sections, based on the trial question about what needs to be measured in: 1) all trials of BS; 2) trials for mucocutaneous involvement; 3) trials for eye involvement; 4) trials for vascular involvement; 5) trials for central nervous system involvement; 6) trials for gastrointestinal involvement and 7) trials for joint involvement.

This article is protected by copyright. All rights reserved 
The questionnaire included an explanation on how the domains in the first section would be assessed in all trials and how in addition to these domains, in the sections on specific organ systems, those domains will only be assessed in trials of that organ or system involvement, according to the trial question. Patients and physicians completed the same questionnaire. Medical terms were explained for the patients. RedCap was used for distribution of the questionnaires and collecting responses in the 2 Delphi rounds. The invitation to participate was sent to 130 patients and 123 physicians. The Turkish version for patients was validated by forward and backwards translation. Items that were agreed on by at least $70 \%$ of either patients or physicians at the end of the first round were included in the questionnaire for the second round of the Delphi.

All of the items were agreed by at least $70 \%$ of the physicians and/or the patients in the first round of the Delphi. Therefore, to decrease the number of domains and subdomains, the participants were asked during the second round of the Delphi to rank the domains that should be assessed in each of the sections. The highest-ranked items in each section were selected without weighting according to the number of patients and experts that responded.

Due to the high level of agreement, it was not necessary to run a third round of the Delphi using the same methods. However, a different approach was utilized due to the specific expertise needed for some of the categories. Lists of the highest-ranked items in the first section (overall disease) and the highest-ranked items in the section that is related to each specialty were sent to experts of that specialty. A total of 37 physician expert in BS were invited, the majority of whom had participated in the first 2 rounds. The experts were asked for their opinion on the mandatory domains that should be assessed in all trials, mandatory domains that should be assessed in trials concerning their specialty, the conditional domains that are important, but not mandatory, and the exploratory domains that could be assessed in specific trials. The responses were discussed among the OMERACT Behçet Syndrome Working Group and the Core Set of domains for clinical trials in BS was created.

\section{OMERACT voting}

Presentation and voting of the Core Set took place during the OMERACT 2018 meeting. Both physicians and patients participated in the voting.

This article is protected by copyright. All rights reserved 


\section{RESULTS}

The following steps were completed and the Core Set of domains were developed, voted upon, and endorsed by the OMERACT community:

\section{Systematic review}

Results of the systematic review were previously published in detail (7). This review explored both the domains of illness studied in BS and the instruments used in research in BS. The instruments chosen for use and emphasis provided insight into the domains of illness felt of primary importance to investigators. The systematic review revealed that 139 outcomes or outcome measures were reported upon in a total of 249 manuscripts. Some of these instruments were specifically developed for BS, such as the Behçet's Disease Current Activity Form (13), the Behçet's Syndrome Activity Scale (14), and the Behçet's Disease Quality of Life Measure (15). Other instruments were non-disease-specific ("generic"), such as the SF36, which are frequently used in other rheumatologic and non-rheumatologic diseases, but were also used in BS trials. The third group of instruments were single-organ measures developed for other diseases that share similar features with BS, such as the Crohn's Disease Activity Index that was used in trials of intestinal involvement of BS (16). However, some of these instruments in the last group have not been validated for use in BS.

Thus, the systematic review showed that there was substantial variability in the domains studied and the outcome measures used for assessing these domains. Despite the large number of outcome measures used in trials of BS, at the time the trial was conducted few were properly validated or widely used. The use of different outcome measures within any one domain, such as disease activity, make it impossible to compare or bring together the results of clinical trials. There was also no standard definition for disease states, such as relapse or remission, or for other key concepts, such as response.

\section{Interest group meeting}

Results of the systematic review were presented to the participants of the expert group and a thorough discussion regarding the difficulties of disease assessment were discussed. The heterogeneity in the clinical presentations of BS, differences in drug response across manifestations, difficulty in defining disease states such as relapse or remission, difficulty in separating disease activity from damage, the inadequacy and lack of instruments to assess patient-important outcomes were the main challenges. Paper cases with different clinical manifestations were presented and "what to measure" in these clinical scenarios was discussed, as were the shortcomings of the available outcome measure instruments.

This article is protected by copyright. All rights reserved 
Suggestions were made on how to develop better instruments. There was general agreement that generic instruments or instruments developed for other diseases could be used as long as they were validated. The group agreed that collaborative work of all stakeholders, including patients with BS, physicians, researchers from all specialties taking care of patients with BS, and representatives from the biopharmaceutical industry, is needed to accomplish the development of a broadly acceptable, datadriven Core Set of outcome measures for BS.

\section{Survey among experts}

A total of 51 experts from different specialties were invited and 35 (69\%) of them responded. The results of this survey were previously reported in detail (2). In summary, the levels of agreement among experts about which domains should be measured in clinical trials of BS were as followed: disease activity (100\% agreement), health-related quality of life (97\%), physical function (83\%), mortality (74\%), disease-related damage (71\%), disease severity (66\%), fatigue (46\%), and overall damage (45\%). Experts were also asked whether they agreed that the four most commonly used disease activity assessment instruments are valid and reliable, with following levels of agreement: Behçet's Disease Activity Index (46\% agreement), the Behçet's Syndrome Activity Scale (43\%), the Clinical Manifestations Index (22\%), and the Iranian Behçet's Disease Dynamic Activity Measure (22\%).

When experts were asked about the necessity of a new instrument for assessing overall disease activity, $89 \%$ agreed that such an instrument is necessary and $97 \%$ agreed that this instrument should include different weighted elements for each clinical manifestation, such as oral ulcers, genital ulcers, other skin lesions, arthritis, uveitis, vascular disease, nervous system lesions, or gastrointestinal lesions. The experts were also asked about the necessity of organ-specific instruments with the following results by organ system: uveitis activity (92\% agreement), neurologic activity (82\%), vascular activity (73\%), oral ulcer activity (73\%), gastrointestinal activity (70\%), genital ulcer activity (59\%), and other cutaneous (papulopustular and nodular lesions) activity (50\%).

\section{Qualitative patient interviews}

The results of the semi-structured qualitative patient interviews were reported in detail elsewhere (9). Several subdomains were identified through these interviews under the 4 main domains, which were symptoms, impact on function, psychological impact and social impact. Skin problems, pain, vision problems, fatigue and sleep disturbances, and gastrointestinal concerns and weight loss were the most common subdomains within the symptom domain. The impact on function could be grouped on the 
categories of impact on speech and vision, mobility, energy for tasks, adaptations, and self-care. Fear, anxiety, stress, depression, and anger were the most frequently discussed emotions in the psychological impact domain. A decreased ability to socialize, negative impact on social duties, especially on family life and work were stressed in the social impact domain. These were useful in identifying patient important domains to be sought for agreement during the Delphi. The data collected through these interviews may also help in developing a BS specific patient reported outcome measure.

\section{Delphi Exercise}

Among the 130 patients and 123 physicians who were invited to participate in the first round of the Delphi, 59 patients (45\%) and 74 physicians (60\%) participated in round 1. Physicians were BS experts from different specialties in 21 countries over 3 continents and most were members of the International Society for Behçet's Disease. Eighty-six percent of the physicians were from academic institutions. Their specialties were rheumatology (50\%), dermatology (16\%), ophthalmology (12\%), internal medicine (12\%), gastroenterology (3\%), and neurology (1\%).

The majority of the patients were from Turkey, Italy, United States, United Kingdom, and France. The clinical manifestations experienced by the patients during their disease course were oral ulcers in $96 \%$, skin lesions in $87 \%$, genital ulcers in $76 \%$, uveitis in $52 \%$, vascular involvement in $44 \%$, nervous system involvement in $32 \%$, and gastrointestinal involvement confirmed by endoscopy in $14 \%$.

All of the 56 domains/subdomains that were sent during Round 1 were endorsed by physicians and/or the patients. All of the domains endorsed by physicians were also endorsed by patients. Additionally, patients endorsed fatigue, sleep, sexual functioning, psychological functioning and acute phase reactants. In order to be able to reduce the number of domains and subdomains to a number that can be feasibly assessed during a trial, the participants were asked to rank the items in the second round of the Delphi. Results of the ranking by physicians and patients for overall assessment and each type of organ involvement are provided in Table 1.

To get a review and validation of the highest-ranked candidate domains in each category, these were sent to experts from the related specialty, together with the overall domains that would be assessed in all trials. Thirty of the 37 experts responded. Among these, 12 were rheumatologists, 5 were ophthalmologists, 5 were dermatologists, 4 were neurologists, 3 were gastroenterologists and 1 was a vascular surgeon. Based on their responses and discussions among the OMERACT Behçet syndrome working group, the Core Set of domains were developed (Figure 1).

This article is protected by copyright. All rights reserved 
The Core Set consists of 5 domains that should be assessed in all BS trials ("mandatory for all trials"). These domains are overall disease activity, new organ involvement, quality of life, adverse events and death. In addition to these there are subdomains that should be assessed in trials for a specific organ involvement ("mandatory per subset"), as follows: i) mucocutaneus: number and pain of lesions; ii) eye: visual acuity, frequency of ocular attacks, ocular severity and vascular leakage; iii) vascular: vascular lesions, superficial thrombophlebitis and post-thrombotic syndrome; iv) central nervous system: central nervous system lesion, cognitive function, and neurologic function; v) gastrointestinal: clinical gastrointestinal activity and endoscopic activity; vi) musculoskeletal: tender joint count and swollen joint count. There are important but optional subdomains that could be assessed according to the purpose of the trial ("optional important domains"); these are mucocutaneous function and duration of lesions for mucocutaneous involvement, ocular damage and cystoid macular edema for eye involvement, and recanalization for vascular involvement. Finally, there are domains and subdomains in the research agenda, including overall function and overall damage for potential use in all trials in BS, pain of arthritis and enthesitis for musculoskeletal involvement, vascular quality of life and inflammatory markers for vascular involvement, and inflammatory markers for gastrointestinal involvement.

\section{OMERACT Voting}

A total of 111 participants who attended the OMERACT 2018 meeting voted. The Core Set was endorsed by $100(90.1 \%)$ of the voters, a remarkably high percentage for any vote at OMERACT, especially for a core set.

This article is protected by copyright. All rights reserved 


\section{DISCUSSION}

The development of a Core Set of domains for use in clinical trials in BS was achieved using the methodology set forth by OMERACT, and through a consensus of patients, physicians, and researchers about "what to measure" in clinical trials for BS. The final Core Set was the result of a multiyear, datadriven, iterative process. The defined domains provide a critical framework for use of outcome measures in BS and as a guide for design of future trials in BS. The domain Core Set will help reduce the heterogeneity of trial designs in BS and harmonize research in this complex disease.

This Core Set of domains includes an important difference from Core Sets previously developed for most other diseases such as rheumatoid arthritis or ANCA-associated vasculitis $(17,18)$. Instead of a single domain set for use in all trials, there is a mandatory set of domains to be used in all trials of BS and separate sets of subdomains specific for each type of organ or system involvement for use in trials seeking to specifically assess that type of involvement. This novel approach to domain selection addresses two key issues: i) a need to generate outcomes data comparable across all trials of BS; and ii) recognition that BS affects many different organ systems that are often studied separately and for which responses to treatments and the treatments themselves may differ. Thus, the proposed Core Set provides a practical framework for harmonizing clinical trial designs in this multisystem disease.

A few examples of the variable disease courses, life impacts, and approaches to treatment are illustrative of the challenges in outcome assessment in BS. Mucocutaneous lesions and joint involvement follow a relapsing and remitting course with symptoms that may impair the quality of life of patients, but do not result in permanent physiologic damage. In contrast, active involvement of the brain, eyes, gastrointestinal tract, or vasculature each carries a risk of long-term damage, organ failure, and in some cases, death. With varying timing, severity, and impact of relapses, current treatment strategies are often quite different for these types of involvement; thus, disease assessment is often different. These variations in course and outcomes in BS have led to the proposed set of core domains for each main organ system. In addition to organ-specific subdomains, it is important to have domains that should be assessed in all trials to avoid missing any new manifestations or the potential impact of an agent in preventing or worsening of those systems not the primary target of the clinical trial.

There were several strengths in the approach to developing this Core Set of domains. The work followed each aspect and the rigorous standards of the OMERACT Filter 2.0 process. The perspectives of patients, physicians, investigators, and methodologists were all strongly taken into consideration with international

This article is protected by copyright. All rights reserved 
representation among each stakeholder group. Additional strengths of the work were the inclusion of patients with each type of organ system involvement in both the qualitative interviews and the Delphi process, and inclusion of experts from all relevant specialties. Consensus and international "buy-in" was reached at each stage and the final Core Set was overwhelmingly endorsed by the OMERACT community.

Some limitations of the project included those inherent in the study of many relatively rare diseases, including potential overrepresentation by stakeholders from one or more regions and relatively small number of participants within each group; however, the study involved participants from multiple countries and several continents.

The proposed approach to domain selection in BS sets a new precedent within OMERACT and provides a paradigm for similar work in other multi-system rheumatic diseases, such as systemic lupus erythematosus and systemic sclerosis (scleroderma), and for diseases in other fields.

Developing this Core Set of domains for trials in BS is an important step in harmonizing clinical trials and data collection in this complex disease with the ultimate aim of enhancing the conduct and comparability of new trials leading to better management and outcomes for patients with BS.

\section{ACKNOWLEDGEMENTS}

The authors thank the many experts in Behçet syndrome who collaborated in the survey and who are listed among the collaborators in the Supplementary Table. The authors also thank the many patients involved in every stage of this project and colleagues with the OMERACT Vasculitis Working Group and the overall OMERACT community for their support and guidance for this project.

This article is protected by copyright. All rights reserved 


\section{REFERENCES}

1. Yazici H, Seyahi E, Hatemi G, Yazici Y. Behçet syndrome: a contemporary view. Nat Rev Rheumatol. 2018 Feb;14(2):107-119.

2. Hatemi G, Ozguler Y, Direskeneli H, Mahr A, Gul A, Levi V, Aydin SZ, Mumcu G, Sertel-Berk O, Stevens RM, Yazici H, Merkel PA. Current Status, Goals, and Research Agenda for Outcome Measures Development in Behçet Syndrome: Report from OMERACT 2014. J Rheumatol. 2015;42(12):2436-41.

3. Ozguler Y, Leccese P, Christensen R, Esatoglu SN, Olivieri I, Yazici H, et al. A Systematic review on the treatment of major organ involvement of Behçet's syndrome informing the EULAR Recommendations for the management of Behçet's Syndrome. Ann Rheum Dis 2016;75(Suppl2): 800.

4. Leccese P, Ozguler Y, Christensen R, Esatoglu SN, Olvieri I, Yazici H, et al. A Systematic review on the treatment of skin, mucosa and joint involvement of Behçet's syndrome informing the EULAR Recommendations for the management of Behçet's Syndrome. Ann Rheum Dis 2016;75(Suppl2): 798.

5. Boers M, Kirwan JR, Wells G, Beaton D, Gossec L, d'Agostino MA, Conaghan PG, Bingham CO 3rd, Brooks P, Landewé R, March L, Simon LS, Singh JA, Strand V, Tugwell P. Developing core outcome measurement sets for clinical trials: OMERACT filter 2.0. J Clin Epidemiol. 2014 Jul;67(7):745-53.

6. Maxwell $\amalg$, Beaton DE, Shea BJ, Wells GA, Boers M, Grosskleg S, et al. Core Domain Set Selection According to OMERACT Filter 2.1: The OMERACT Methodology. J Rheumatol. 2019; 46 (8) 1014-1020.

7. Hatemi G, Merkel PA, Hamuryudan V, Boers M, Direskeneli H, Aydin SZ, et al. Outcome measures used in clinical trials for Behçet syndrome: a systematic review. J Rheumatol 2014 Mar;41:599-612.

8. Hatemi G, Meara A, Ozguler Y, Direskeneli H, Mahr A, Easley E, Gurcan M, Davis T, Gul A, Yazici Y, Zottenberg K, Esatoglu SN, Erer B, Kamali S, Yazici H, Cronholm PF, and Merkel 
PA. Developing a Core Set of Outcome Measures for Behçet Disease: Report from OMERACT 2016. J Rheumatol 2017 44: 1750-1753.

9. Ozguler Y, Merkel PA, Gurcan M, Bocage C, Eriksen W, Kutlubay Z, Hatemi G, Cronholm PF; OMERACT Behçet's Syndrome Working Group. Patients' experiences with Behçet's syndrome: structured interviews among patients with different types of organ involvement. Clin Exp Rheumatol. 2019;37 Suppl 121(6):28-34.

10. Glaser BG. The Constant Comparative Method of Qualitative Analysis. Social Problems. $1965 ; 12(4): 436-445$.

11. Boeije H. A Purposeful Approach to the Constant Comparative Method in the Analysis of Qualitative Interviews. Quality and Quantity. 2002;V36(4):391.

12. Boers M, Kirwan JR, Wells G, Beaton D, Gossec L, d'Agostino MA, et al. Developing core outcome measurement sets for clinical trials: OMERACT filter 2.0. J Clin Epidemiol 2014;67:745-53.

13. Lawton G, Bhakta BB, Chamberlain MA, Tennant A. The Behcet's disease activity index. Rheumatology (Oxford). 2004;43(1):73-8.

14. Forbess C, Swearingen C, Yazici Y. Behçet's Syndrome Activity Score (BSAS): a new disease activity assessment tool, composed of patient-derived measures only, is strongly correlated with the Behçet's Disease Current Activity Form (BDCAF) Arthritis Rheum. 2008;58(Suppl 9):S854.

15. BD Gilworth G, Chamberlain MA, Bhakta B, Haskard D, Silman A, Tennant A. Development of the BD-QoL: a quality of life measure specific to Behçet's disease. J Rheumatol. 2004;31:931-937.

16. Best WR, Becktel JM, Singleton JW, Kern F Jr. Development of a Crohn's disease activity index. National cooperative Crohn's disease study. Gastroenterology 1976; 70: 439-44.

17. Boers M, Tugwell P, Felson DT, van Riel PL, Kirwan JR, Edmonds JP, Smolen JS, Khaltaev N, Muirden KD: World Health Organization and International League of Associations for Rheumatology core endpoints for symptom modifying antirheumatic drugs in rheumatoid arthritis clinical trials. J Rheumatol. 1994, 21 (suppl 41): 86-89. 
18. Merkel PA, Aydin SZ, Boers M, Direskeneli H, Herlyn K, Seo P, Suppiah R, Tomasson G, Luqmani RA. The OMERACT core set of outcome measures for use in clinical trials of ANCA-associated vasculitis. J Rheumatol. 2011;38(7):1480-6.

Figure Legend

Figure 1. Core Set of Domains for Study in Clinical Trials of Behçet Syndrome 
Table 1. Physicians' and patients' rank order of importance of domains and subdomains for inclusion in clinical trials for each category of disease manifestation for Behçet syndrome.

\begin{tabular}{|c|c|c|}
\hline Physicians & Patients & Physicians and Patients* \\
\hline \multicolumn{3}{|l|}{ Overall } \\
\hline Overall BS disease activity & Overall BS disease severity & Overall BS disease activity \\
\hline $\begin{array}{l}\text { Flare of existing organ } \\
\text { involvement }\end{array}$ & Damage & $\begin{array}{l}\text { Flare of existing organ } \\
\text { involvement }\end{array}$ \\
\hline New organ involvement & New organ involvement & Overall BS disease severity \\
\hline Overall BS disease severity & $\begin{array}{l}\text { Flare of existing organ } \\
\text { involvement }\end{array}$ & New organ involvement \\
\hline Overall function & Overall BS disease activity & Overall function \\
\hline Physician global assessment of BS & Quality of life & Damage \\
\hline Patient global assessment of BS & Overall function, & Quality of life \\
\hline Quality of life & Psychological well being & Patient global assessment of BS \\
\hline Damage & Patient global assessment of BS & Physician global assessment of BS \\
\hline Acute phase response & Fatigue & Psychological well being \\
\hline Fatigue & Acute phase response & Fatigue \\
\hline Psychological well being & Physician global assessment of BS & Acute phase response \\
\hline \multicolumn{3}{|l|}{ Skin and mucosa involvement } \\
\hline Mucocutaneous activity & Pain of oral ulcers & Mucocutaneous activity \\
\hline Mucocutaneous severity & Mucocutaneous activity & Pain of oral ulcers \\
\hline Number of oral ulcers & Number of oral ulcers & Number of oral ulcers \\
\hline Pain of oral ulcers & Pain of genital ulcers & Mucocutaneous severity \\
\hline Number of genital ulcers & Mucocutaneous severity & Pain of genital ulcers \\
\hline Pain of genital ulcers & Pain of nodular lesions & Number of genital ulcers \\
\hline
\end{tabular}

This article is protected by copyright. All rights reserved 


\begin{tabular}{|c|c|c|}
\hline Physicians & Patients & Physicians and Patients* \\
\hline Mucocutaneous function & Number of genital ulcers & Mucocutaneous function \\
\hline Number of nodular lesions & Mucocutaneous function & Pain of nodular lesions \\
\hline Pain of nodular lesions & Number of papulopustular lesions & Number of nodular lesions \\
\hline Number of papulopustular lesions & Number of nodular lesions & Number of papulopustular lesions \\
\hline \multicolumn{3}{|l|}{ Joint involvement } \\
\hline Joint involvement activity & Joint involvement severity & Joint involvement activity \\
\hline Swollen joint count & Pain & Joint involvement severity \\
\hline Tender joint count & Joint involvement activity & Swollen joint count \\
\hline Joint involvement severity & Tender joint count & Tender joint count \\
\hline Pain & Swollen joint count & Pain \\
\hline \multicolumn{3}{|l|}{ Eye involvement } \\
\hline Ocular involvement activity & Ocular involvement severity & Ocular involvement activity \\
\hline Visual acuity & Visual acuity & Visual acuity \\
\hline Ocular involvement severity & Ocular involvement activity & Ocular involvement severity \\
\hline Retinal vasculitis & Retinal vasculitis & Retinal vasculitis \\
\hline Number of ocular attacks & Retinal infiltrates & Number of ocular attacks \\
\hline Retinal infiltrates & Capillary leak & Retinal infiltrates \\
\hline Cystoid macular edema & Number of ocular attacks & Cystoid macular edema \\
\hline Glucocorticoid cessation/tapering & Cystoid macular edema & Capillary leak \\
\hline Capillary leak & Glucocorticoid cessation/tapering & Glucocorticoid cessation/tapering \\
\hline \multicolumn{3}{|l|}{ Vascular involvement } \\
\hline New/extending venous thrombus & New arterial aneurysm & New arterial aneurysm \\
\hline New arterial thrombus & New arterial thrombus & New/extending venous thrombus \\
\hline New arterial aneurysm & New/extending venous thrombus & New arterial thrombus \\
\hline Superficial thrombophlebitis & Superficial thrombophlebitis & Superficial thrombophlebitis \\
\hline
\end{tabular}

This article is protected by copyright. All rights reserved 


\begin{tabular}{|c|c|c|}
\hline Physicians & Patients & Physicians and Patients* \\
\hline \multicolumn{3}{|l|}{ Central nervous system involvement } \\
\hline New/Flare of existing involvement & New/Flare of existing involvement & New/Flare of existing involvement \\
\hline Progression on MRI & Progression on MRI & Progression on MRI \\
\hline Cognitive functioning & Mood disorders & Cognitive functioning \\
\hline Headache & Headache & Headache \\
\hline Mood disorders & Cognitive functioning & Mood disorders \\
\hline \multicolumn{3}{|l|}{ Gastrointestinal involvement } \\
\hline Gastrointestinal activity & Flare of existing involvement & Gastrointestinal activity \\
\hline Flare of existing involvement & Gastrointestinal activity & Flare of existing involvement \\
\hline Abdominal pain & Perforation/surgery & Perforation/surgery \\
\hline Perforation/surgery & Diarrhea & Abdominal pain \\
\hline Diarrhea & Hematochezia & Diarrhea \\
\hline Hematochezia & Weight loss & Hematochezia \\
\hline Weight loss & Hematemesis & Weight loss \\
\hline Hematemesis & Abdominal pain & Hematemesis \\
\hline Nausea & Nausea & Nausea \\
\hline
\end{tabular}

BS: Behçet syndrome. ${ }^{*}$ The order of the items in the third column was arrived at by combining the preferences of physicians and patients.

This article is protected by copyright. All rights reserved 


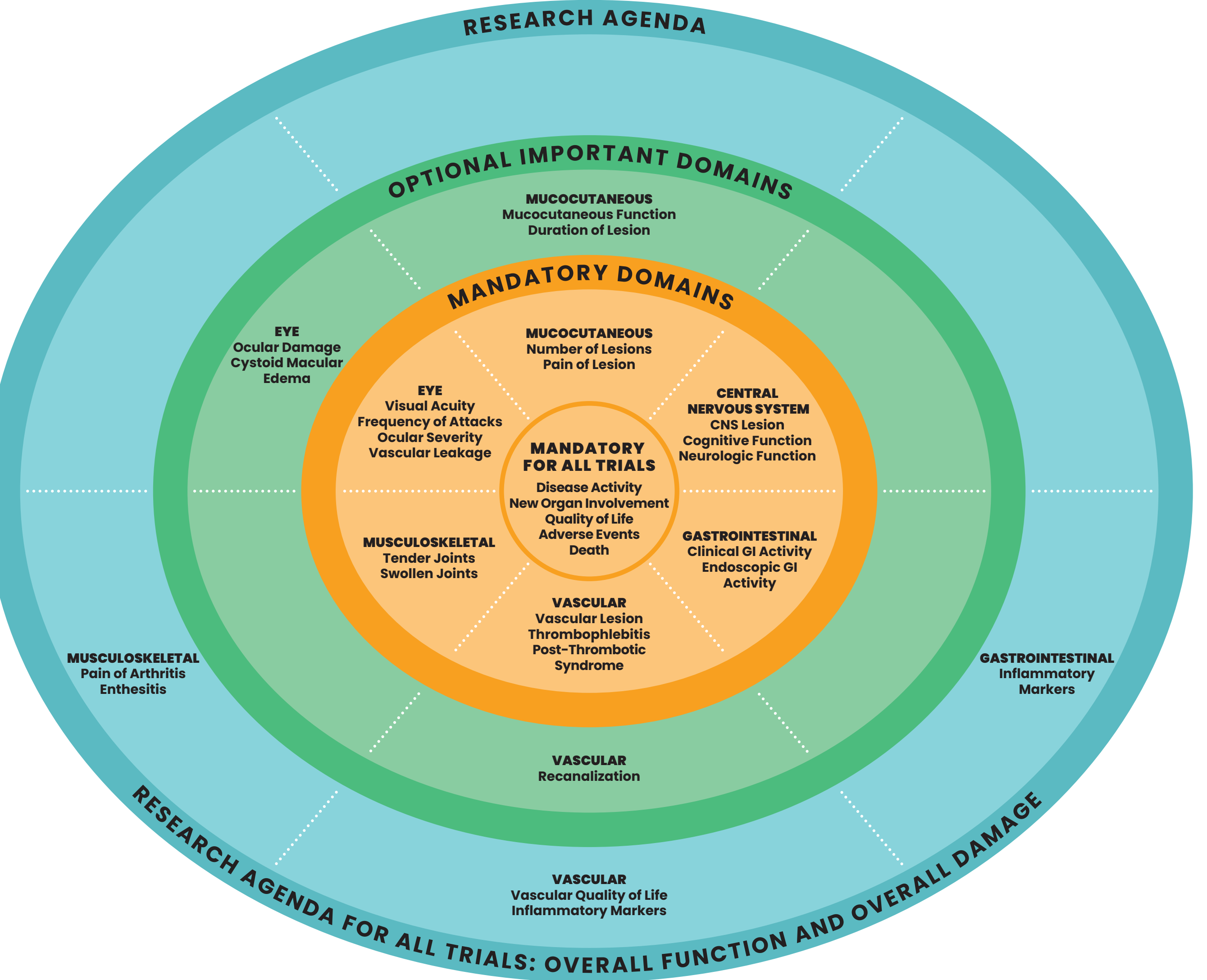

